\title{
Short Pulse Laser Applications Design
}

R. P. J. Town, D. S. Clark, A. J. Kemp, B. F. Lasinski, and M. Tabak

February 11, 2008

Final Report LDRD 07-ERD-040 


\section{Disclaimer}

This document was prepared as an account of work sponsored by an agency of the United States government. Neither the United States government nor Lawrence Livermore National Security, LLC, nor any of their employees makes any warranty, expressed or implied, or assumes any legal liability or responsibility for the accuracy, completeness, or usefulness of any information, apparatus, product, or process disclosed, or represents that its use would not infringe privately owned rights. Reference herein to any specific commercial product, process, or service by trade name, trademark, manufacturer, or otherwise does not necessarily constitute or imply its endorsement, recommendation, or favoring by the United States government or Lawrence Livermore National Security, LLC. The views and opinions of authors expressed herein do not necessarily state or reflect those of the United States government or Lawrence Livermore National Security, LLC, and shall not be used for advertising or product endorsement purposes.

\section{Auspices Statement}

This work performed under the auspices of the U.S. Department of Energy by Lawrence Livermore National Laboratory under Contract DE-AC52-07NA27344. This work was funded by the Laboratory Directed Research and Development Program at LLNL under project tracking code 07-ERD-040. 
LLNL-TR-401411

Final Report LDRD 07-ERD-040

\title{
Short Pulse Laser Applications Design*
}

\author{
R. P. J. Town, D. S. Clark, A. J. Kemp, B. F. Lasinski, and M. Tabak \\ Lawrence Livermore National Laboratory, Livermore, CA 94551-0808, USA
}

\begin{abstract}
We are applying our recently developed, LDRD-funded computational simulation tool to optimize and develop applications of Fast Ignition (FI) for stockpile stewardship. This report summarizes the work performed during a one-year exploratory research LDRD to develop FI point designs for the National Ignition Facility (NIF). These results were sufficiently encouraging to propose successfully a strategic initiative LDRD to design and perform the definitive FI experiment on the NIF.
\end{abstract}

\section{Introduction/Background}

Ignition experiments on the National Ignition Facility (NIF) will begin in 2010 using the central hot spot (CHS) approach, which relies on the simultaneous compression and ignition of a spherical fuel capsule. Unlike this approach, the fast ignition (FI) method separates fuel compression from the ignition phase. In the compression phase, a laser such as NIF is used to implode a shell either directly, or by x rays generated from the hohlraum wall, to form a compact dense $\left(\sim 300 \mathrm{~g} / \mathrm{cm}^{3}\right)$ fuel mass with an areal density of $\sim 3.0 \mathrm{~g} / \mathrm{cm}^{2}$. To ignite such a fuel assembly requires depositing $\sim 20 \mathrm{~kJ}$ into a $\sim 35 \mu \mathrm{m}$ spot delivered in a short time compared to the fuel disassembly time ( $\sim 20 \mathrm{ps})$. This energy is delivered during the ignition phase by relativistic electrons generated by the interaction of an ultra-short high-intensity laser.

The main advantages of FI over the CHS approach are higher gain, a lower ignition threshold, and a relaxation of the stringent symmetry requirements required by the CHS approach.

There is worldwide interest in FI and its associated science. Major experimental facilities are being constructed which will enable "proof of principle" tests of FI in integrated subignition experiments, most notably the OMEGA-EP facility at the University of Rochester's Laboratory of Laser Energetics and the FIREX facility at Osaka University in Japan. Also, scientists in the European Union have recently proposed the construction of a new FI facility, called HiPER, designed to demonstrate FI. Our design work has focused on the NIF, which is the only facility capable of forming a full-scale hydro assembly, and could be adapted for full-scale FI by the conversion of additional beams to short-pulse operation.

\footnotetext{
* This work performed under the auspices of the U.S. Department of Energy by Lawrence Livermore National Laboratory under Contract DE-AC52-07NA27344. This work was funded by the Laboratory Directed Research and Development Program at LLNL under project tracking code 07-ERD-040.
} 


\section{Research Activities}

This section briefly reviews the work that has been performed on two key areas to develop a NIF FI point design, namely hydrodynamic implosion and calculations of the hot electron conversion efficiency and temperature distribution. The widely differing spatial, temporal, and density scales needed to accurately model the FI process leads to a computationally challenging project that is difficult to solve using one code employing a single solution technique. We have ntegrated different physics codes (radiationhydrodynamics, explicit and implicit particle-in-cell (PIC) codes) using the PYTHON programming language to form a virtual code.

Optimized isochoric hydro implosion configuration at NIF-scale.

Most inertial confinement fusion (ICF) implosion designs involve the formation of a low density, high entropy core. In fact, the formation of this region is crucial for the CHS ignition approach, but for FI the presence of the hotspot leads to a lower efficiency design. We have developed an analytic implosion model that carefully tailors the fuel adiabat in flight to minimize the residual hot spot. This technique has been applied to a direct-drive design that used $485 \mathrm{~kJ}$ of blue laser light in a 30 -ns highly shaped laser pulse to drive a cryogenic capsule. The capsule design consists of a thin $\mathrm{CH}$ overcoat shell that contained a $180 \mu \mathrm{m} 30 \mathrm{mg} / \mathrm{cm}^{3} \mathrm{CH}$ foam wicked with DT (the ablator) and a $230 \mu \mathrm{m}$ clean DT fuel layer. The stagnated density profile taken from a one-dimensional HYDRA simulation around peak compression is shown in figure 1 . The residual hotspot is extremely compact and the fuel density is in excess of $200 \mathrm{~g} / \mathrm{cm}^{3}$ for $\sim 200 \mathrm{ps}$. The maximum areal density of this design was $3.1 \mathrm{~g} / \mathrm{cm}^{2}$.

The NIF will be used initially in an indirect-drive configuration and so we subsequently developed a range of indirect-drive designs. To convert to an indirect-drive design, a Beryllium-copper ablator replaced the wicked foam ablator, although alternatives using high-density carbon and doped plastic give similar 1-D performance. Shown in figure 2 is

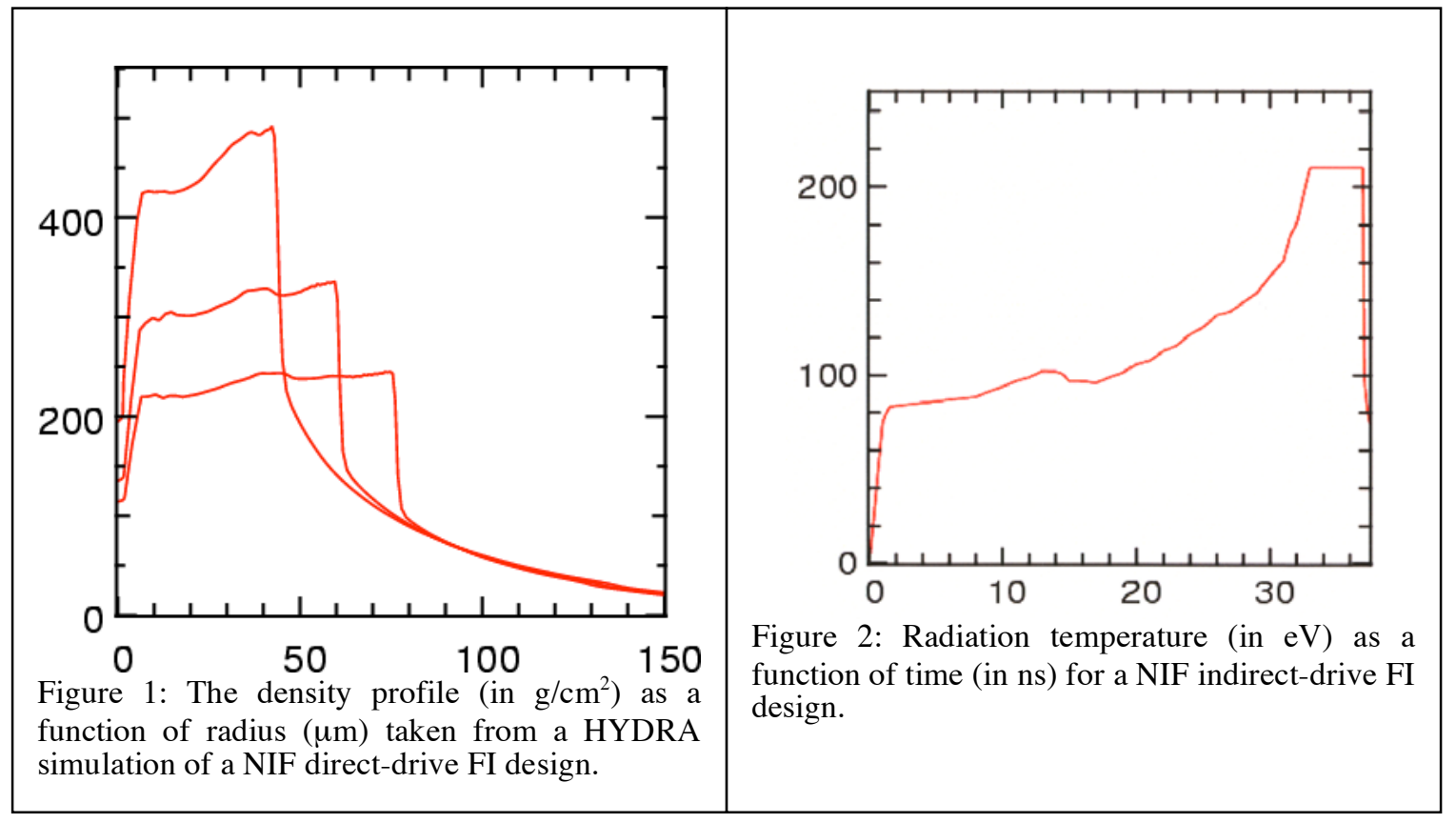


the radiation drive history that was used to compress the fuel on a low adiabat $\left(\alpha_{\text {stagnation }} \sim\right.$ 1.2) to densities $\sim 300 \mathrm{~g} / \mathrm{cm}^{3}$. This design required $\sim 800 \mathrm{~kJ}$ of $\mathrm{x}$ rays to compress the fuel and would require about $10 \mathrm{~kJ}$ of hot electrons to yield 56MJ. However, the hydrodynamic efficiency of this design is low.
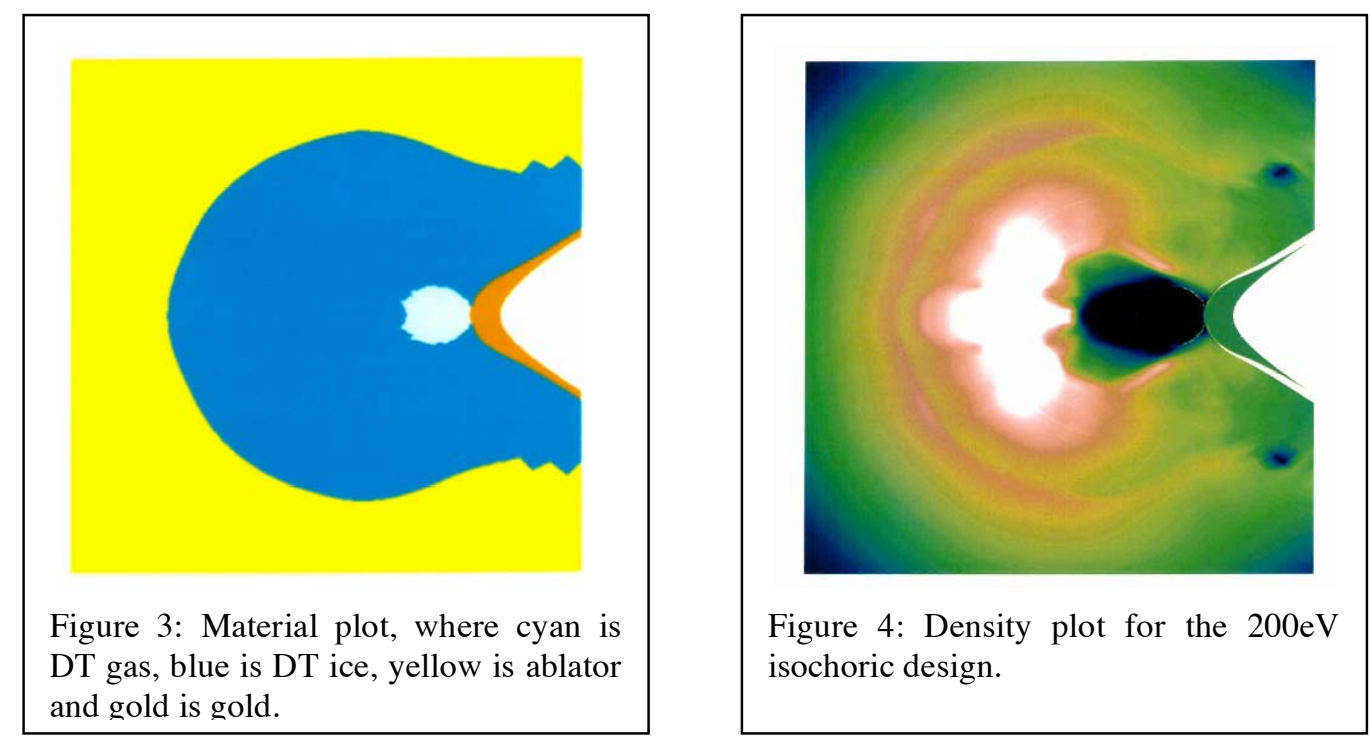

In order to minimize the hot electron transport distance from their generation by the short-pulse laser (at the relativistic critical surface) to the compressed fuel most FI designs embed a gold cone into the capsule. This is designed to leave a clear path for the short-pulse laser to propagate through up to the cone tip that is placed close to the center of the capsule. The presence of the cone introduces a large asymmetry in the implosion that we have started to model in 2-D. Figure 3 shows a material plot around peak compression for the isochoric $200 \mathrm{eV}$ design. The residual DT gas is confined to a very small volume and the gold cone has been compressed by the shells passage. Figure 4 shows a density contour map which shows the formation of a high density fuel configuration, which is located around $100 \mu \mathrm{m}$ from the tip of the gold cone.

\section{Hot electron conversion efficiency and energy.}

To ignite the hydro design discussed above requires about $20 \mathrm{~kJ}$ of hot electron energy. To deliver that energy in a $\sim 35 \mu \mathrm{m}$ spot in about $\sim 20$ ps requires a laser intensity of $\sim 4 \times 10^{20} \mathrm{~W} / \mathrm{cm}^{2}$ (assuming a conversion efficiency of $25 \%$ ). However, the hot electrons need an energy of $\sim 2 \mathrm{MeV}$ to reach the compressed fuel. If the pondermotive scaling law holds then the intensity of the short-pulse laser would be $2 \times 10^{19} \mathrm{~W} / \mathrm{cm}^{2}$. If we can improve the coupling efficiency and tailor the density profile then the short-pulse laser energy and intensity requirements can be decreased.

We have performed 1- and 2-D particle-in-cell (PIC) calculations to examine the conversion efficiency and hot electron energy distribution function when the short-pulse laser interacts with a planar target. When the laser interacts with a density profile the laser steepens the profile, which leads to a reduction in the laser-generated hot-electron temperature. Figure 5 shows the initial density profile $\left(\mathrm{n} \sim \mathrm{e}^{(\mathrm{x} / 0.65)}\right)$ and the profile obtained when an $\mathrm{a}_{0}=20$ laser interacts with the plasma after $200 \mathrm{fs}$. The initial $3 \mu \mathrm{m}$ 


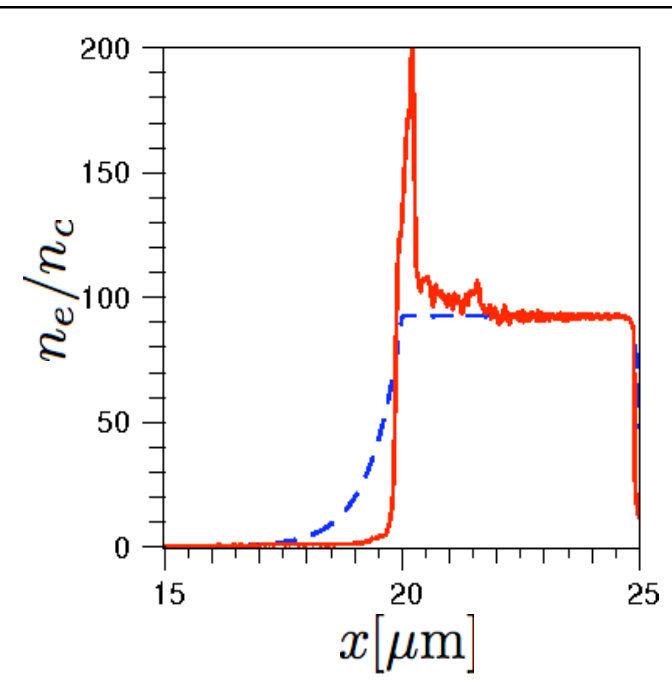

Figure 5: The density profile initially (blue dotted line) and after the laser has interacted for $200 \mathrm{fs}$ (red line).

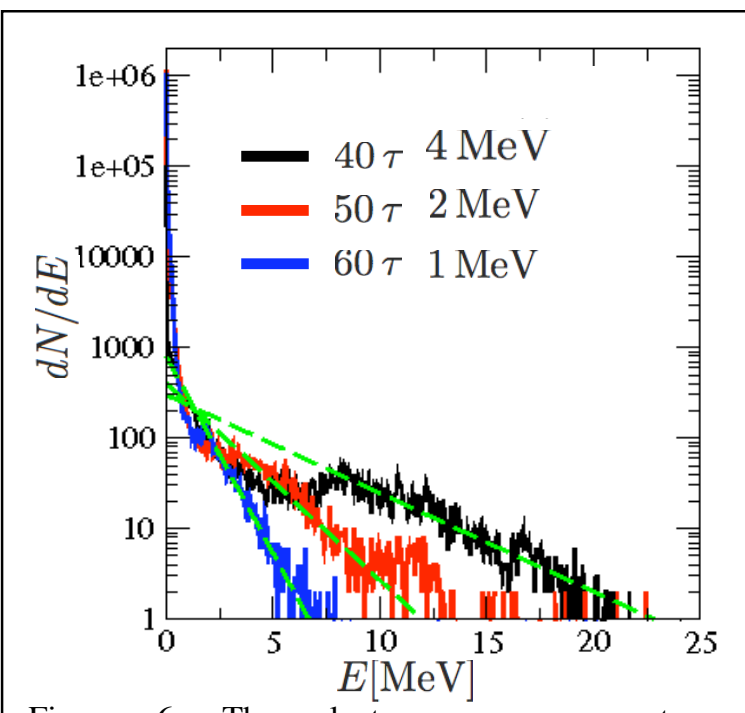

Figure 6: The electron energy spectrum temperature reduces at later times as the density profile is modified by the interaction.

density profile has steepened leaving a low-density shelf in front of the target, which leads to a reduction in the hot electron temperature at later times as shown in figure 6.

In the FI design the laser will interact with a cone rather than a planar foil and so we have performed 2D PIC simulations of cones. These simulations have shown that cones have higher coupling efficiency into hot electrons and a higher hot electron temperature than planar targets. For example, the hot electron temperature rose from $0.9 \mathrm{MeV}$ to $1.6 \mathrm{MeV}$ when a $10^{19} \mathrm{~W} / \mathrm{cm}^{2}$ laser was incident on a pointed cone compared to a planar target. We have also examined the effect of roughened surfaces on the conversion efficiency. We obtained 55\% absorption when the laser was incident on a $4 \mu \mathrm{m}$ deep grooved surface compared to $30 \%$ for a flat surface. The absorption increased to $65 \%$ when the groove depth was doubled. However, the hot electrons appeared in a $30^{\circ}$ cone angle and these results will need to be confirmed for pulses longer than the $0.5 \mathrm{ps}$ simulations reported here.

\section{Exit Plan}

This LDRD developed the design groundwork to enable early FI demonstration experiments on the NIF. The design work performed in the first year was sufficiently encouraging to successfully propose a strategic initiative LDRD to design and perform the definitive FI experiment on the NIF.

\section{Summary}

We are applying our recently developed, LDRD-funded computational simulation tool to optimize and develop applications of FI for stockpile stewardship. This report has summarized the work performed during a one-year exploratory research LDRD to develop FI point designs for the NIF. 


\section{A. Publications and Presentations Resulting From This LDRD}

1. D. S. Clark and M. Tabak “A Self-Similar Isochoric Implosion for Fast Ignition”, Nuc. Fusion 47, 1147 (2007) UCRL-JRNL-229868.

2. A. J. Kemp, et al., "Laser heating of solid matter by light pressure-driven shocks at ultra-relativistic intensities", Anomalous Absorption Conference, Maui, HI, 27-31 August 2007. UCRL-PRES-233896.

3. B. F. Lasinski, et al., "PIC Simulations of Short-Pulse, High-Intensity Light Impinging on Structured Targets", Fast Ignition Workshop, Cambridge MA, 3-5 November 2006. UCRL-PRES-225535.

4. M. Tabak, et al., "Hydrodynamic issues for Fast Ignition", APS Division of Plasma Physics, Philadelphia, PA 20 October - 3 November 2006. UCRL-POST225428.

5. R. P. J. Town, et al., "Integrated Modeling if Fast Ignition Experiments", Fast Ignition Workshop, Cambridge MA, 3-5 November 2006. UCRL-ABS-222479.

6. R. P. J. Town, et al., "Status of Fast Ignition Point Designs for the NIF", Fifth International Conference on Inertial Fusion Sciences and Applications, Kobe, Japan, 9-14 September 2007. UCRL-ABS-232204. 